\title{
Benzoyl Transfer for Footprinting Alcohol-Containing Residues in Higher Order Structural Applications of Mass Spectrometry Based Proteomics
}

Austin B. Moyle, Ming Cheng*†, Nicole D. Wagner, Michael L. Gross*

Department of Chemistry, Washington University in St. Louis, St. Louis, MO 63130 United States

†Present address: Center of Technology, Transitional Science, BioMarin Pharmaceuticals, San Rafael, CA 94901, United States

Corresponding authors' email addresses: mgross@wustl.edu, ming.cheng@wustl.edu

\section{Table of Contents}

Experimental Methods.

2

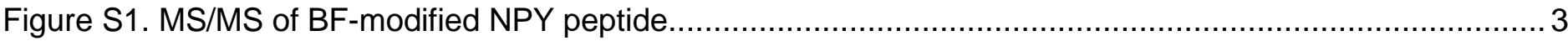

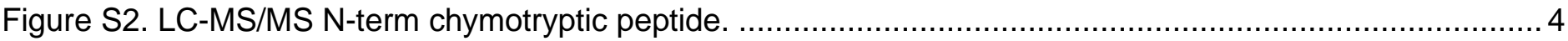

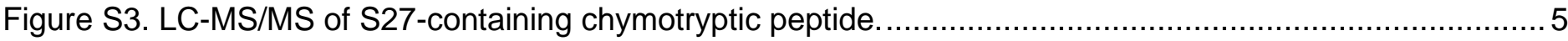

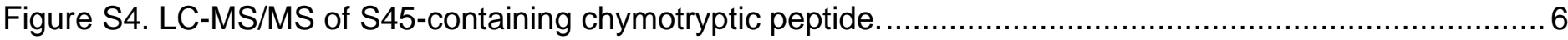

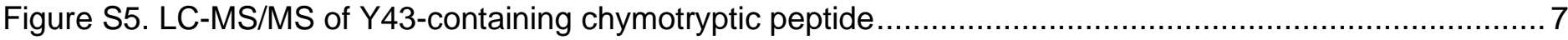

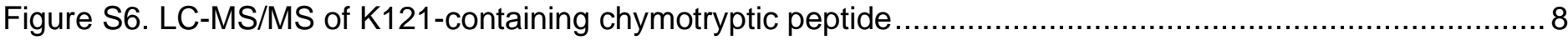

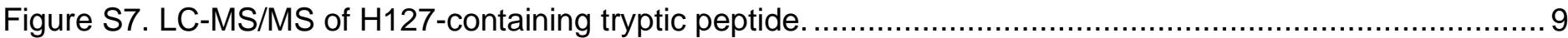

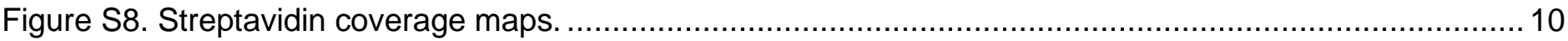

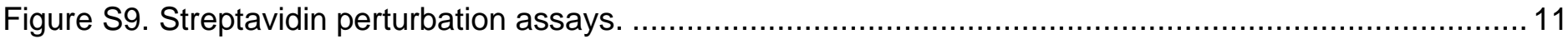

Figure S10. Intact Analysis of modified and BF-modified streptavidin. ................................................ 12

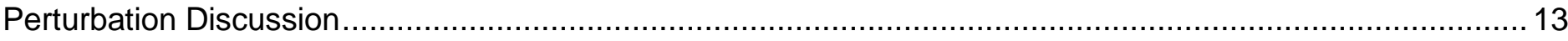

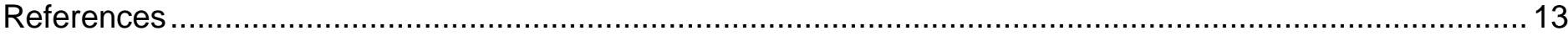




\section{Experimental Methods}

Materials. Unless otherwise indicated, all reagents were sourced from Millipore Sigma (St. Louis, MO) and used without further purification. Neuropeptide $Y$ (18-36) was dissolved in water and diluted in PBS to a concentration of $10 \mu \mathrm{M}$. Streptavidin was dissolved in water and diluted in PBS to a concentration of $5 \mu \mathrm{M}$ tetramer. For biotinbound state, $20 \mu \mathrm{M}$ biotin was added and incubated with streptavidin overnight. Benzoyl fluoride (TCl Chemicals, Tokyo, Japan) was diluted with acetonitrile to a stock concentration of $100 \mathrm{mM}$.

Benzoylation of NPY (18-36). NPY was incubated with a range of molar equivalents to peptide-10, 50, 100, 500 in excess-at $25^{\circ} \mathrm{C}$ and shaken at 200rpm before LC-MS desalting and analysis.

Benzoylation and DEPC modification of c(RGDYE). c(RGDYE) (Peptides International, Louisville, KY, USA) was dissolved in water and diluted in a phosphate buffered saline solution (PBS, $\mathrm{pH} 7.4$ unless otherwise indicated) to a concentration of $10 \mu \mathrm{M}$. The BF-modifications of cyclopeptides for kinetic evaluation were completed at various BF concentrations and incubated for 2 min at $37^{\circ} \mathrm{C}$ with $200 \mathrm{rpm}$ agitation. Cysteine quench ( 50 equivalents with respect to the highest BF concentration) was used to quench the reaction, and the samples were flash frozen for later LC-MS analysis. DEPC-modification of cyclic peptides for kinetic evaluation was completed at various DEPC concentrations and incubated for 2 min at $37^{\circ} \mathrm{C}$ with $200 \mathrm{rpm}$ agitation. Aqueous imidazole (Oakwood Chemical, Estill, SC) ( 50 equivalents with respect to the highest DEPC concentration) were used to quench the reaction, and the samples were flash frozen for later LC-MS analysis. All samples for kinetics analyses were prepared and analyzed in at least duplicate.

Benzoylation of Streptavidin-Biotin. Apo and holo streptavidin (13-139) were incubated in 500 molar equivalents of excess BF for $5 \mathrm{~min}$ at $25^{\circ} \mathrm{C}, 200 \mathrm{rpm}$ agitation. The BF reaction was quenched by removal of the BF reagent (Zeba Spin Desalting Columns, $7 \mathrm{k} \mathrm{MWCO}$, Thermo Fisher, Waltham, MA). The modified streptavidin was denatured at $90^{\circ} \mathrm{C}$ for $10 \mathrm{~min}$. To the resulting denatured streptavidin solution, Tris buffer was added (final $=100 \mathrm{mM}, \mathrm{pH}=8.0$ ), followed by overnight chymotrypsin digestion $(1: 20, \mathrm{w} / \mathrm{w})$ (Thermo Fisher).

LC-MS Analyses. For LC-MS analysis, the modified NPY peptide and C(RGYDE) samples were trapped, desalted, and eluted from a C18 trap (Opti-guard $1 \mathrm{~mm}$ C18 guard column, Millipore Sigma, St. Louis, MO) and analyzed using a maXis 4G Q-ToF (Bruker Daltonics, Billerica, MA). All LC-MS data of peptides were compiled and analyzed by using Bruker Data Analysis 4.2 software. For LC-MS analysis of unmodified and BF-labeled streptavidin, samples were trapped, desalted, and eluted from a C4 column (ACQUITY UPLC Protein BEH C4 Column, $300 \AA, 1.7 \mu \mathrm{m}, 2.1 \mathrm{~mm}$ X $50 \mathrm{~mm}$, Waters Corporation, Milford MA). Data were compiled and analyzed using Intact ${ }^{\mathrm{TM}}$ (Protein Metrics Inc, Cupertino, California).

LC-MS/MS Analyses. For LC-MS/MS analyses of the labeled streptavidin-biotin peptides, the modified peptides were trapped and desalted on an custom packed C18 trap desalting column on an Dionex Ultimate 3000 (Thermo Fisher) and eluted into a Q Exactive Plus Orbitrap MS (Thermo Scientific, Waltham, Germany) via nano-ESI, as described earlier ${ }^{1}$. The LC-MS/MS data for streptavidin digest peptides were analyzed using Byonic ${ }^{\mathrm{TM}}$ and Byologic $\AA$ (Protein Metrics Inc., Cupertino, California) as previously described ${ }^{1}$. Unmodified and BF-modified peptides (+104.0262 Da) were compared for apo and holo streptavidin. Statistical significance of modification differences were determined by a Student's two-tailed t-test.

Far UV Circular Dichroism. Samples were run on a CD Spectrometer (Chirascan, Applied Photophysics, Skipton, UK) and analyzed on Chirascan Pro-Data Viewer software. Samples were analyzed in a cuvette with a $2 \mathrm{~mm}$ pathlength, measured at $2 \mathrm{~s}$ per time point from 250-350 nm with $1 \mathrm{~nm}$ step size, $1 \mathrm{~nm}$ bandwidth, with three repeats each, and spectra converted to mean residue molar ellipticity.

Fluorescence Melting Point Assay. Samples were run on a FP-8300 Spectrofluorometer (JASCO, Oklahoma City, OK) with Xe lamp with the settings: emission mode, $5 \mathrm{~nm}$ Ex bandwidth, $5 \mathrm{~nm}$ Em bandwidth, $50 \mathrm{msec}$ response, manual sensitivity, 300 V PMT voltage, $300-400 \mathrm{~nm}$ measurement range, $1 \mathrm{~nm}$ data interval, 280.0 $\mathrm{nm}$ excitation wavelength, scan speed $100 \mathrm{~nm} / \mathrm{min}$. Across T range $25-80{ }^{\circ} \mathrm{C}, 5^{\circ} \mathrm{C}$ interval, ramp rate of 10 ${ }^{\circ} \mathrm{C} / \mathrm{min}$.

\section{Dynamic Light Scattering}

Samples were analyzed on a DynaPro-PlateReader II (Wyatt Technologies Corporation, Goleta, CA). Measurements were acquired in at least duplicate, obtained at $25^{\circ} \mathrm{C}$, and analyzed with Dynamics software (Wyatt). 
A

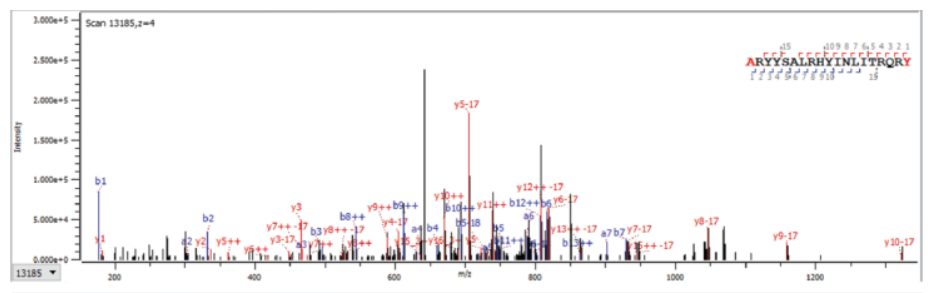

B

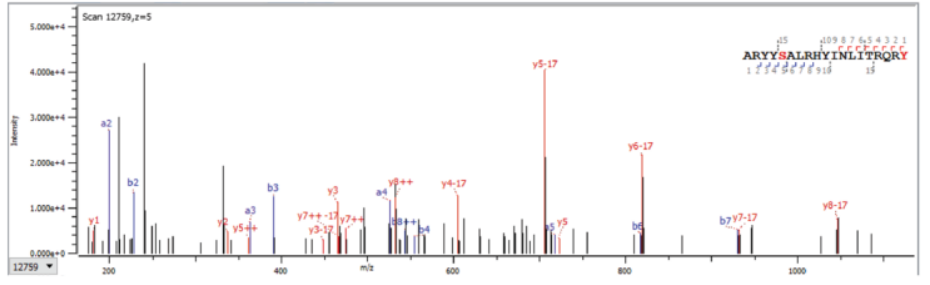

C

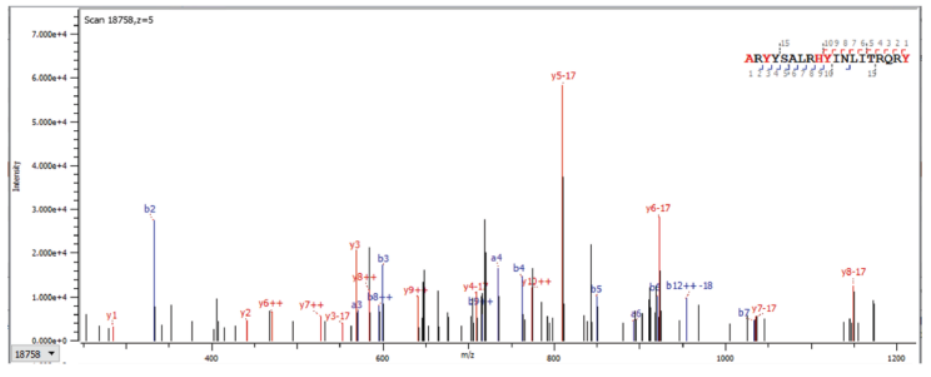

D

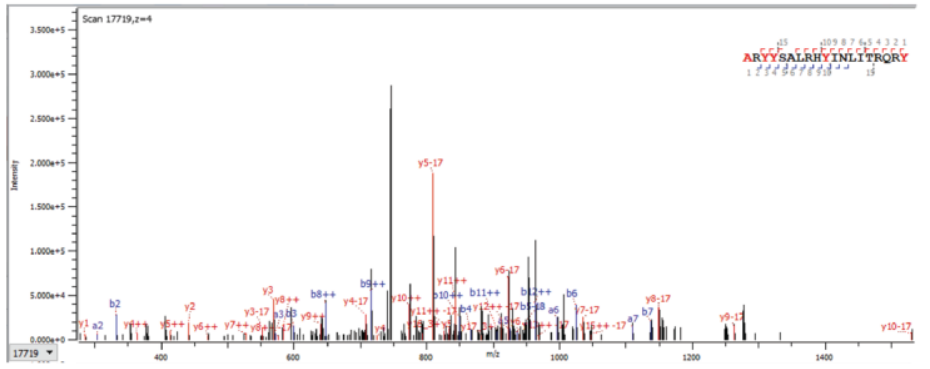

E

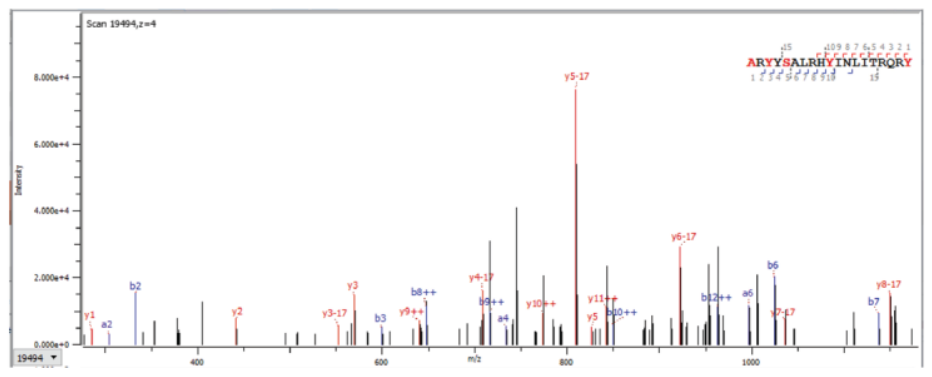

Figure S1. MS/MS of BF-modified NPY peptide.

Representative MS/MS spectra of NPY peptide fragments suggesting C-terminal amidation and BF modification of N-terminus (A) and Ser5 (B); BF modification of N-terminus, Tyr3, His9, Tyr10, and Tyr19 (C); BF modification of N-terminus, Tyr3, Tyr4, Tyr19 (D); BF modification of N-terminus, Tyr3, Ser5, Tyr10, and Tyr19 (E). 

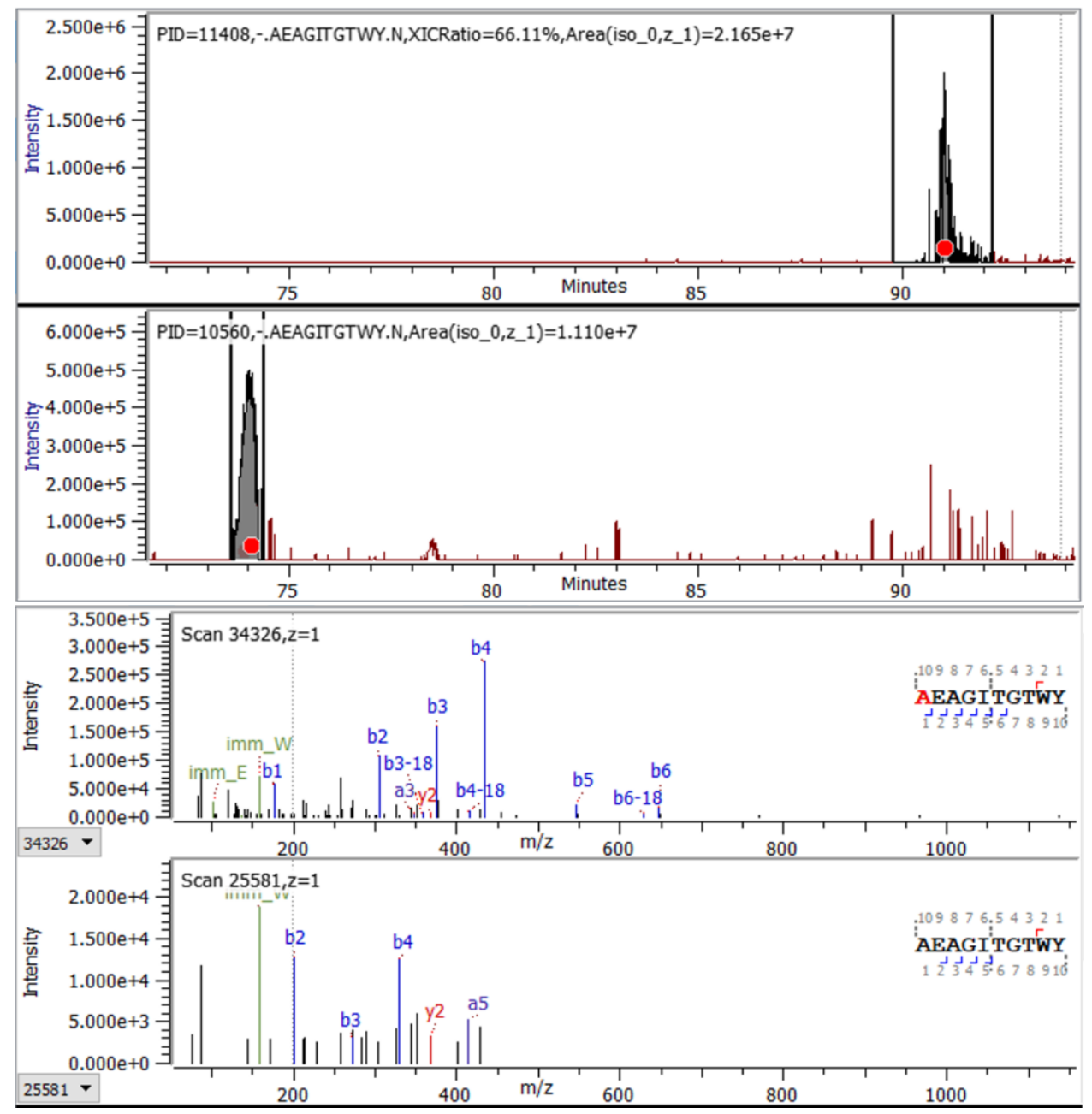

Figure S2. LC-MS/MS N-term chymotryptic peptide.

Extracted ion chromatogram (XIC) of BF-modified and unmodified peptide (top) eluting at $\sim 74$ min and $\sim 91$ min, respectively. Product-ion (MS/MS) spectrum of BF-modified and unmodified peptide (bottom) identifying $\mathrm{N}$-term as the site of BF modification. 

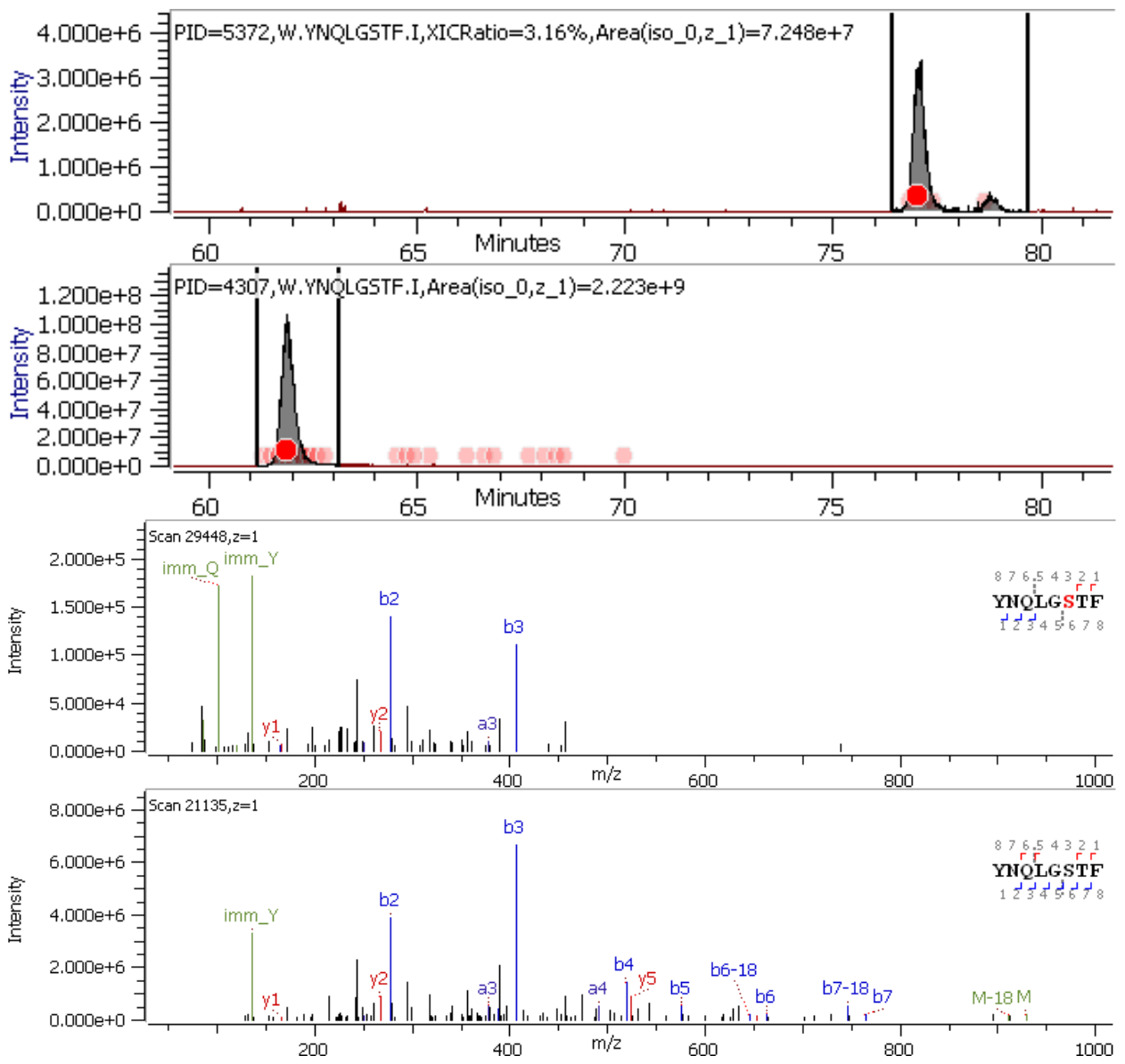

Figure S3. LC-MS/MS of S27-containing chymotryptic peptide.

Extracted ion chromatogram (XIC) of BF-modified and unmodified peptide (top) eluting at $\sim 77$ min and $~ 62$ min, respectively. Product-ion (MS/MS) spectrum of BF-modified and unmodified peptide (bottom) identifying S27 as the site of BF modification. 

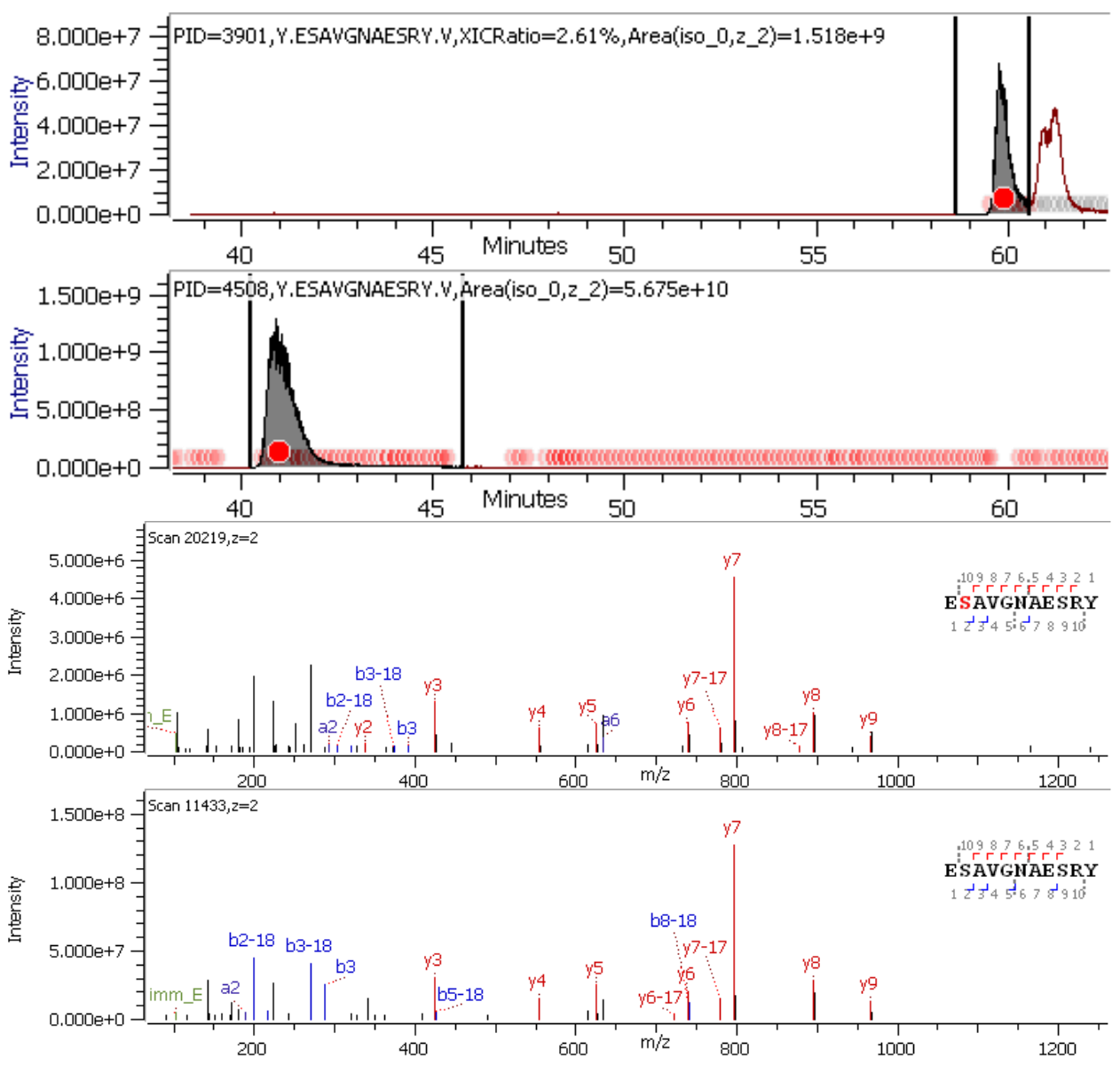

Figure S4. LC-MS/MS of S45-containing chymotryptic peptide.

Extracted ion chromatogram (XIC) of BF-modified and unmodified peptide (top) eluting at $\sim 60$ min and 41 min, respectively. Product-ion (MS/MS) spectrum of BF-modified and unmodified peptide (bottom) identifying S45 as the site of BF modification. 

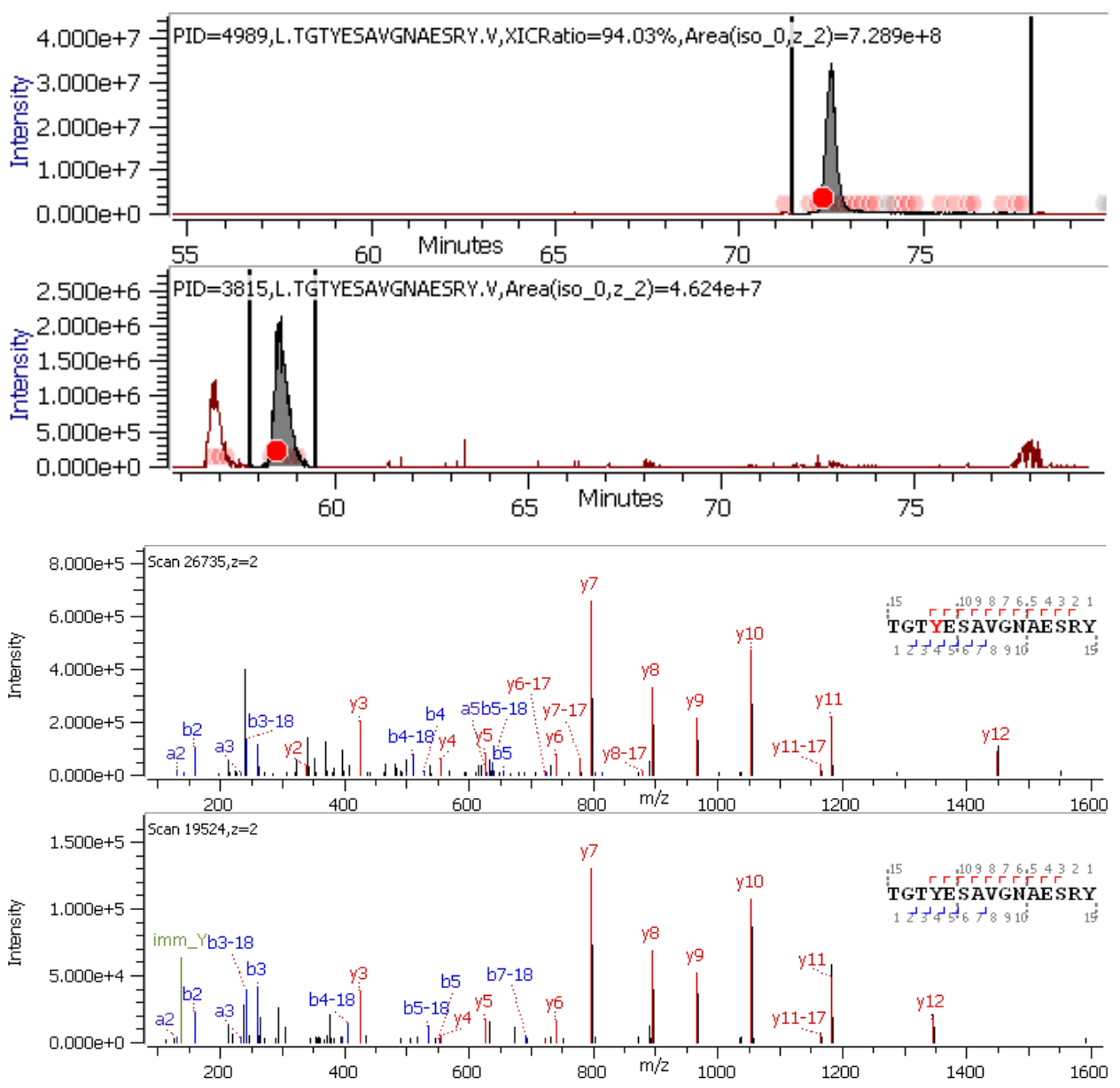

Figure S5. LC-MS/MS of Y43-containing chymotryptic peptide

Extracted ion chromatogram (XIC) of BF-modified and unmodified peptide (top) eluting at $\sim 72.5$ min and $~ 58.5$ min, respectively. Product-ion (MS/MS) spectrum of BF-modified and unmodified peptide (bottom) identifying Y43 as the site of BF modification. 

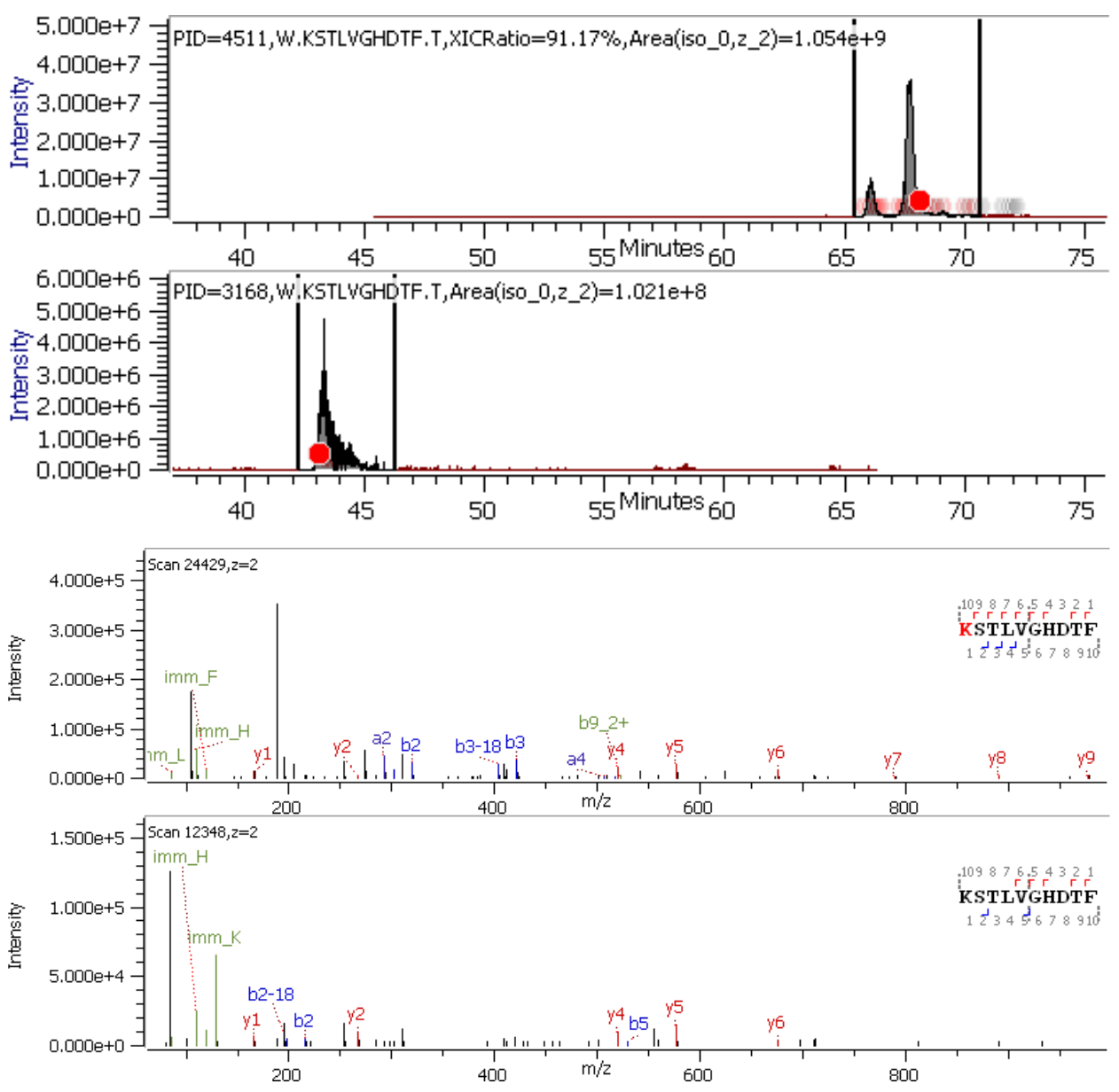

Figure S6. LC-MS/MS of K121-containing chymotryptic peptide

Extracted ion chromatogram (XIC) of BF-modified and unmodified peptide (top) eluting at $\sim 68$ min and $\sim 43$ $\mathrm{min}$, respectively. Product-ion (MS/MS) spectrum of BF-modified and unmodified peptide (bottom) identifying $\mathrm{K} 121$ as the site of BF modification. 

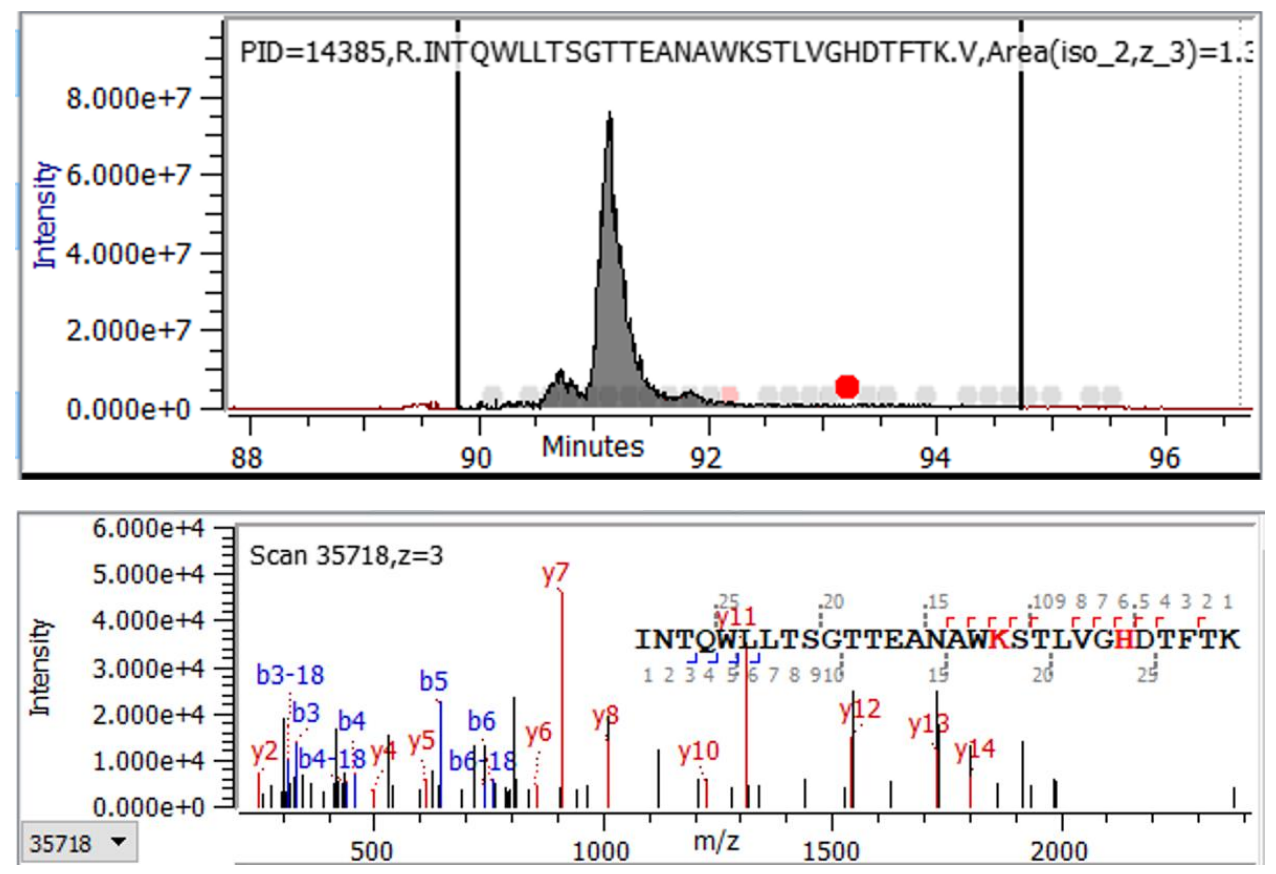

Figure S7. LC-MS/MS of H127-containing tryptic peptide.

Extracted ion chromatogram (XIC) of double BF-modified peptides (top) eluting at $93 \mathrm{~min}$. Double modification of specifically $\mathrm{K}$ and $\mathrm{H}$ appears low in relative abundance to other doubly modified species.Product-ion (MS/MS) spectrum of BF-modified peptide (bottom) identifying H127 as a site of BF modification. Unmodified peptide was not observed. 

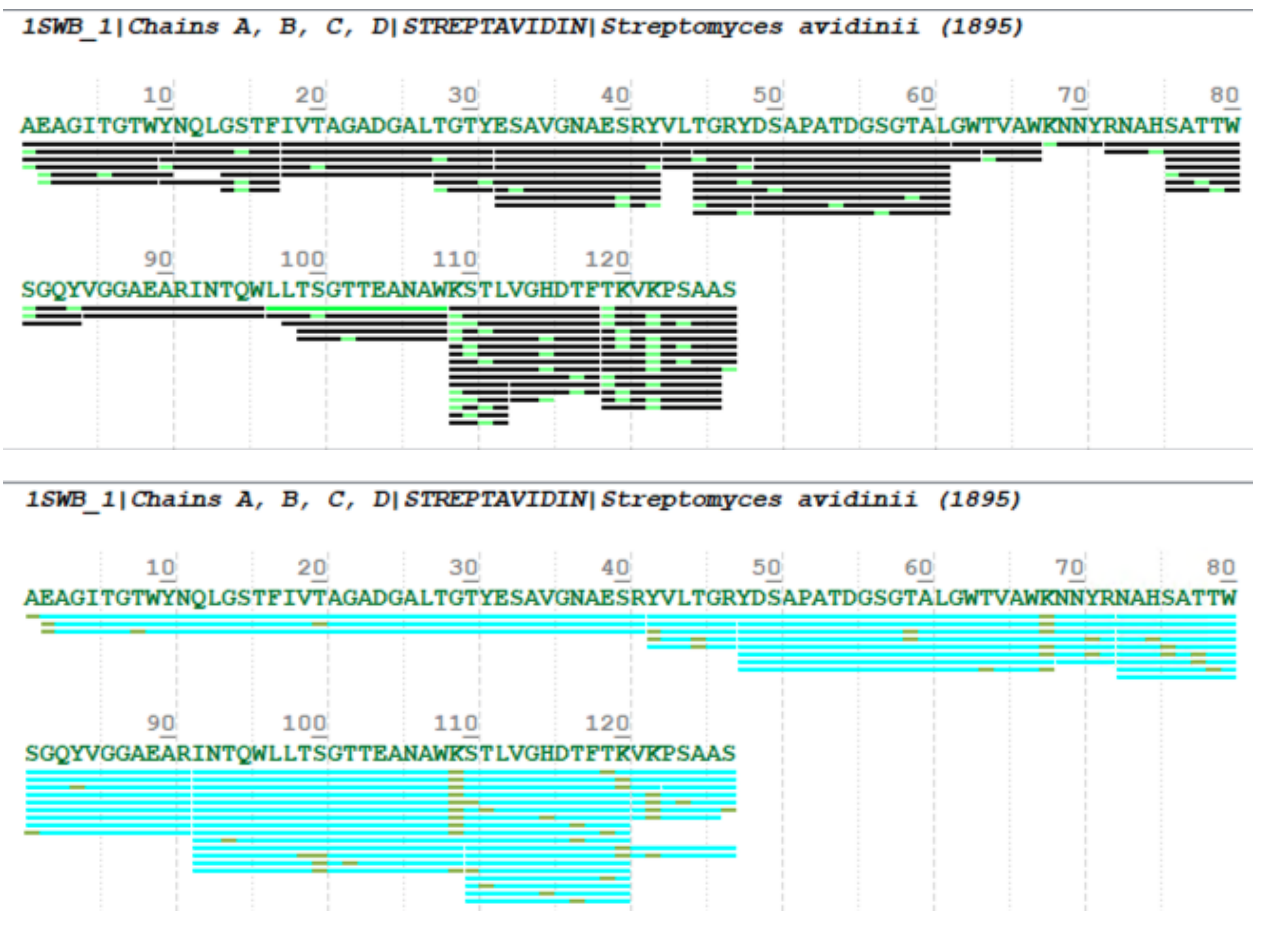

Figure S8. Streptavidin coverage maps.

Chymotrypsin (top, black) and trypsin (bottom, blue) digested streptavidin (13-139) and submitted to LCMS/MS analysis. Green highlights in the peptides indicate sites of BF modification identified by the software, but not all peptides are used in Figure 3 for evaluation of biotin binding site. Our data indicate that a mutation, A116E (here residue 104), is the predominant species of this commercial protein, which is further supported by exact measurements of intact protein in Figure S7. 
A

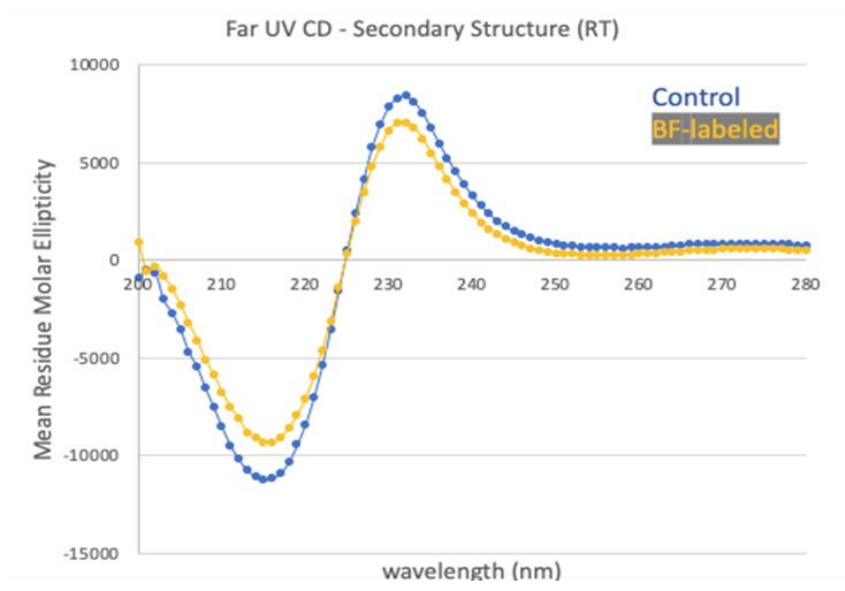

C

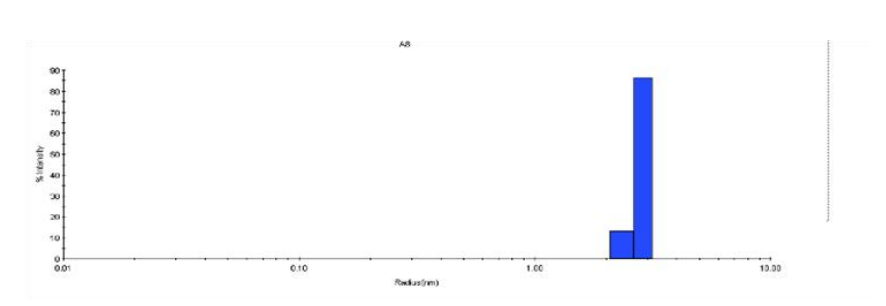

B

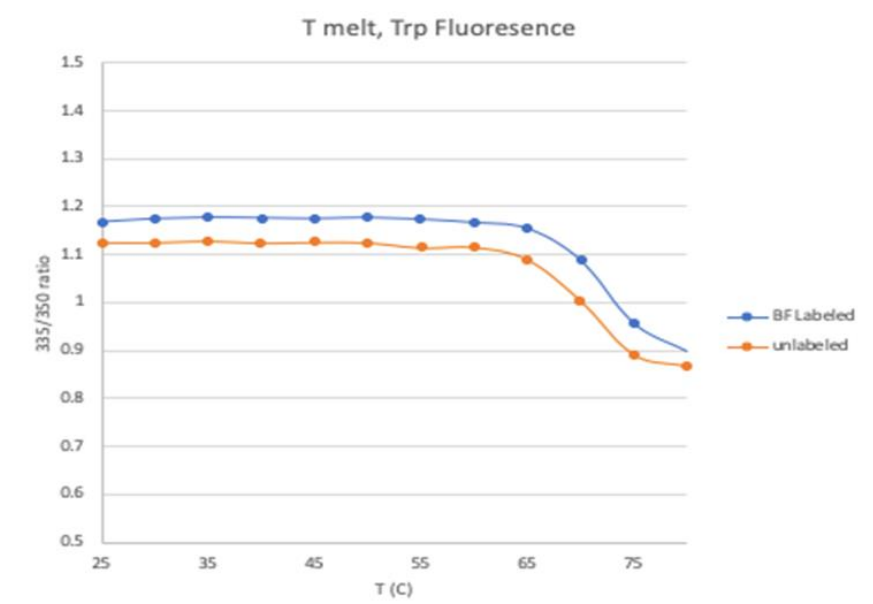

D

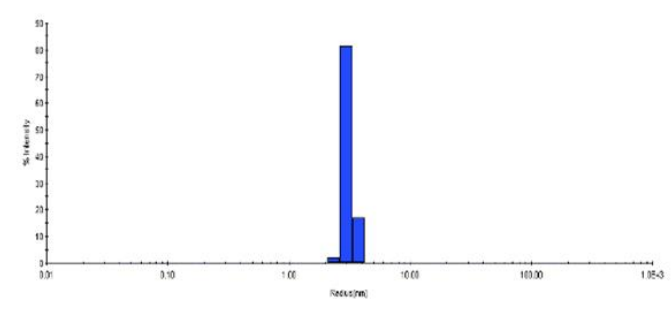

Figure S9. Streptavidin perturbation assays.

(A) Far UV CD spectrum where the $215 / 233 \mathrm{~nm}$ intensity ratio of BF-labeled streptavidin has a difference of $1.2 \%$ compared to control (i.e., secondary structure unperturbed). (B) Trp fluorescence melt monitoring $335 / 350 \mathrm{~nm}$ intensity ratios align with control (i.e., tertiary structure unperturbed). Dynamic light scattering of unlabeled (C) and BF-modified streptavidin (D), yield insignificant differences in hydrodynamic radii $(3.0 \pm 0.2$ $\mathrm{nm}$ and $3.2 \pm 0.1 \mathrm{~nm}$, respectively) with polydispersity $<15 \%$, indicating a homogenous quaternary structure of homotetramer is maintained upon labeling. 


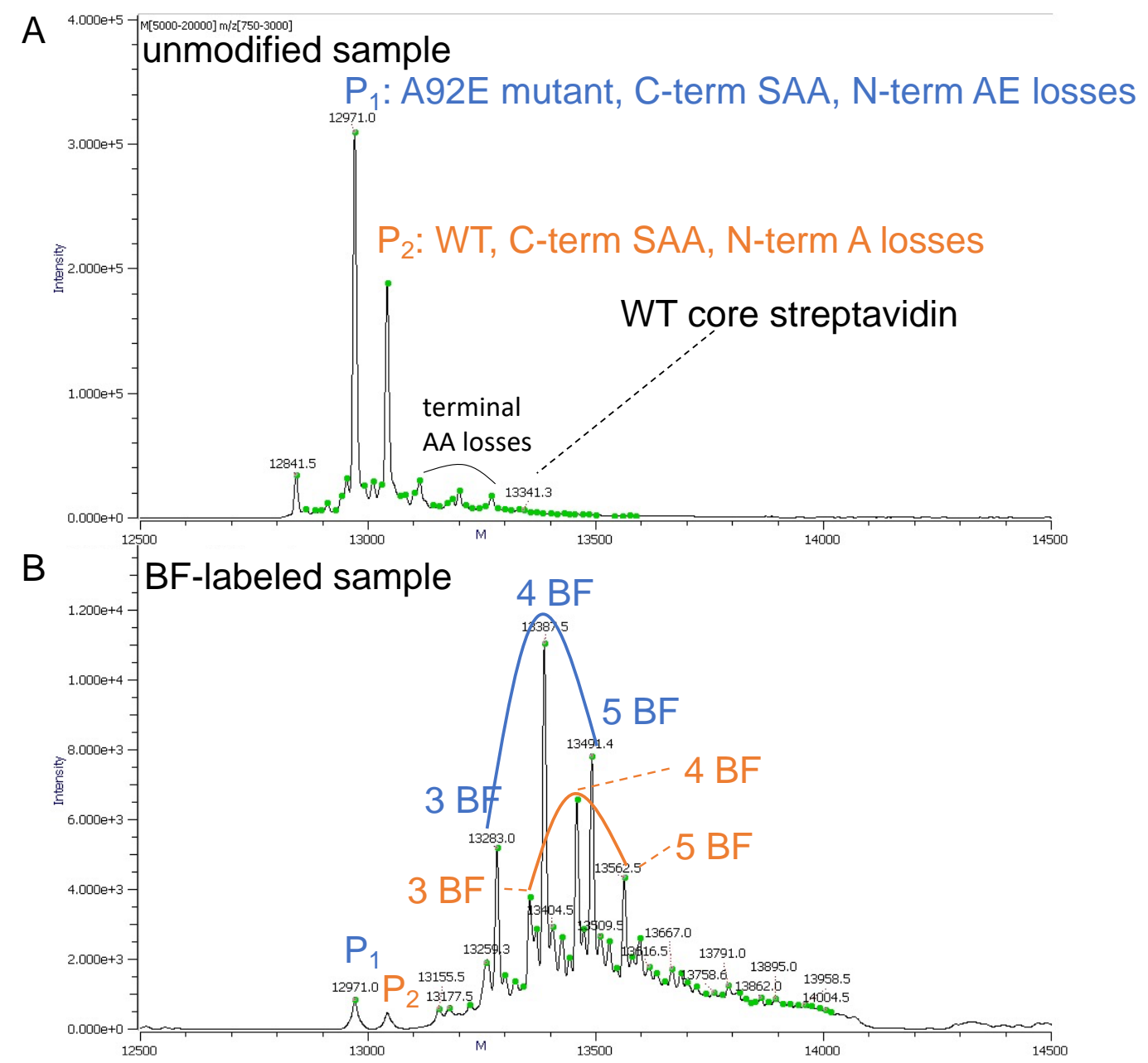

Figure S10. Intact Analysis of modified and BF-modified streptavidin.

(A) Deconvoluted mass spectrum of unmodified streptavidin, indicating terminal amino acid (AA) losses for both wildtype protein (WT: $P_{2}$, orange) and an A116E mutant protein ( $P_{1}$, blue) present in the commercial protein sample. The dominant $A 116 \mathrm{E}$ mutant is also validated by bottom-up analysis in Figure S5. (B) BF modifications of $\mathrm{P}_{1}$ (blue) and $\mathrm{P}_{2}$ (orange), indicating an average of four BF modifications per protein. 


\section{Structure Perturbation Discussion}

The high concentrations of labelling reagents necessary for footprinting can potentially induce structural perturbations to the protein. Experiments with labeling reagents, especially those with labelling timescales in minutes or longer, necessitate improved measurements to ensure that the reagent or modification is not perturbing HOS. This can be accomplished by looking for kinetic distortions, whereby global protein modification is measured as a function of reagent concentration ${ }^{2}$; upon structural perturbation by the footprinting, the protein will presumably become more solvent-exposed and the reactivity will break upward from linearity. Gross and coworkers $^{3}$ argue that a protein has maintained HOS integrity so long as the distribution of modified states for a given charge state is Poisson. Others have used functional assays or other techniques including circular dichroism ${ }^{1}, \mathrm{UV}$-Vis, or kinetics if the protein is an enzyme ${ }^{4}$. Here, we argue that the appropriateness for various assays is a function of the protein system. For BF evaluation, we applied far-UV circular dichroism (CD), Trp fluorescence monitored melting, and dynamic light scattering to measure changes in secondary, tertiary, and quaternary structure, respectively.

Far-UV Circular Dichroism. CD spectra of unmodified streptavidin (Figure S6A) are consistent with previously recorded spectra for the wild type streptavidin tetramer ${ }^{5}$. Upon BF labeling, the CD spectra undergo minimal change. Differences observed in the far UV region may be due to subtle differences in concentration; indeed, the labeled sample contains $5 \%$ organic $\mathrm{V} / \mathrm{V}$, which may be lowered to $1 \% \mathrm{~V} / \mathrm{V}$ for future experiments. Further, the relative magnitude of the absolute minima (at $215 \mathrm{~nm}$ ) and maxima (at $233 \mathrm{~nm}$ ) in the spectra are found for the wild type and footprinted samples (1.36 and 1.38 for labeled and unlabeled, respectively), indicating that significant secondary structural perturbations do not occur (the difference in magnitude is less than $1.2 \%$ ).

Fluorescence melting point assay. Streptavidin binds to biotin via a hydrophilic, Trp-rich pocket, and Trp fluorescence was previously reported to be sensitive to streptavidin structure ${ }^{6}$. Changes in unlabeled Trp side chain orientation can be probed by fluorescence to see whether tertiary HOS is maintained. The probe indicates that BF modification does not affect the heat-induced unfolding of a streptavidin (Figure S6B). The shift in $335 / 350$ for both labeled and unlabeled streptavidin at $70^{\circ} \mathrm{C}$ is comparable, indicating that the responses of the modified and unmodified protein are comparable, indicating maintenance of structure upon reaction with BF.

Dynamic light scattering. Given that streptavidin is a $55 \mathrm{kDa}$ homotetramer, quaternary structural perturbation is measurable by dynamic light scattering, utilizing its $0.1 \mathrm{~nm}$ resolution. Our DLS measurements yield no significant differences in spherical radius of unmodified vs. modified streptavidin $(3.0 \pm 0.2 \mathrm{~nm}$ and $3.15 \pm 0.09$ $\mathrm{nm}$ (Figures S6C and S6D, respectively). Of course, dynamic light scattering (DLS) also probes the oligomerization state by measuring the hydrodynamic radius. ${ }^{7}$ Further, polydispersity exhibited by both states is within the $15 \%$ limit for significant differences, indicating single populations of labeled and unlabeled streptavidin. Taking all the evidence together, BF footprinting does not perturb the HOS of streptavidin on the time scale of our experiments.

\section{References}

(1) Cheng, M.; Asuru, A.; Kiselar, J.; Mathai, G.; Chance, M. R.; Gross, M. L. Fast Protein Footprinting by X-ray Mediated Radical Trifluoromethylation. Journal of the American Society for Mass Spectrometry 2020. DOI: 10.1021/jasms.0c00085. (2) Limpikirati, P. K.; Zhao, B.; Pan, X.; Eyles, S. J.; Vachet, R. W. Covalent Labeling/Mass Spectrometry of Monoclonal Antibodies with Diethylpyrocarbonate: Reaction Kinetics for Ensuring Protein Structural Integrity. Journal of the American Society for Mass Spectrometry 2020. DOI: 10.1021/jasms.0c00067.

(3) Gau, B. C.; Sharp, J. S.; Rempel, D. L.; Gross, M. L. Fast Photochemical Oxidation of Protein Footprints Faster than Protein Unfolding. Analytical Chemistry 2009, 81 (16), 6563-6571. DOI: 10.1021/ac901054w.

(4) Cheng, M.; Guo, C.; Li, W.; Gross, M. L. Free-Radical Membrane Protein Footprinting by Photolysis of Perfluoroisopropyl lodide Partitioned to Detergent Micelle by Sonication. Angew Chem Int Ed Engl 2021, 60 (16), 8867 8873. DOI: 10.1002/anie.202014096 From NLM.

(5) Kuo, t.; Lee, P.-C.; Tsai, C.-W.; Chen, W.-Y. Salt bridge exchange binding mechanism between streptavidin and its DNA aptamer - Thermodynamics and spectroscopic evidences. Journal of molecular recognition : JMR 2013, 26, 149159. DOI: $10.1002 / j m r .2260$.

(6) Waner, M. J.; Hiznay, J. M.; Mustovich, A. T.; Patton, W.; Ponyik, C.; Mascotti, D. P. Streptavidin cooperative allosterism upon binding biotin observed by differential changes in intrinsic fluorescence. Biochem Biophys Rep 2019, 17, 127-131. DOI: 10.1016/j.bbrep.2018.12.011 PubMed.

(7) Su, Z.; Wu, C.; Shi, L.; Luthra, P.; Pintilie, G. D.; Johnson, B.; Porter, J. R.; Ge, P.; Chen, M.; Liu, G.; et al. Electron Cryo-microscopy Structure of Ebola Virus Nucleoprotein Reveals a Mechanism for Nucleocapsid-like Assembly. Cell 2018, 172 (5), 966-978.e912. DOI: 10.1016/j.cell.2018.02.009 PubMed. 